\title{
Two-Soliton Solutions of Relativistic Field Equations
}

\author{
O. Steinmann
}

Fakultät für Physik, Universität Bielefeld, D-4800 Bielefeld 1, Federal Republic of Germany

\begin{abstract}
Existence of solutions converging for $t \rightarrow-\infty$ to a superposition of two solitons is shown for a class of scalar, relativistic, field equations in two-dimensional space-time.
\end{abstract}

\section{Introduction}

Recently there has been considerable interest in the possible relevance of soliton solutions of classical field equations for the description of elementary particles (see e.g. the reviews [1-3]). By "solitons" we understand here static solutions with finite energy of a relativistic field equation, and the time dependent solutions obtained from them by Lorentz transformation. Note that this definition, which has become accepted in particle physics, is more general than the one used in the mathematical literature as reviewed by Scott et. al. [4].

The energy of a soliton remains essentially localized in some finite, nonexpanding region for all times. This feature survives quantization, at least for the models and in the approximations so far investigated [1-3]. But this is the behaviour expected from an extended particle, and the question comes up naturally, whether some of the particles observed in nature may be described with the help of soliton solutions. Obviously, such an application is meaningful only if it is possible to introduce states with more than one particle. This presupposes the existence of solutions of the field equation in question, which can be interpreted as describing the movement of several solitons.

Such several-soliton solutions are explicitly known for the sine-Gordon equation $[5,6]$. They are solutions which are, for large positive or negative time, approximate superpositions of well separated solitons moving with different velocities. Number, form, and velocities, are the same for $t \rightarrow-\infty$ as for $t \rightarrow \infty$ : the solitons undergo time delay but no genuine scattering. Arguments have been adduced [7] that solutions of this type may also exist in the $\varphi_{2}^{4}$ kink model. The persistence property just mentioned is an essential part of the original definition of solitons. However, interesting though equations of this type may be from the mathematical point of view, they are clearly of limited value for applications to particle physics: realistic particles do scatter.

We must therefore widen our field of inquiry. Any relativistic field equation 
possessing one-soliton solutions in the general meaning introduced above, is a candidate for investigation. We define a $n$-soliton solution of such an equation as a solution which converges for $t \rightarrow-\infty$ in a suitable topology towards a superposition of $n$ spatially separated solitons, whilst nothing is assumed about the behaviour at $t \rightarrow+\infty$. More exactly, such a solution should be called an incoming $n$-soliton solution. Outgoing solutions are defined analogously by prescribing the $t \rightarrow+\infty$ asymptotics. In this paper we shall only consider in-solutions. Our proofs and results are, of course, easily transcribed to the out-case.

Little seems to be known about the existence of $n$-soliton solutions in the general case. The present paper is a step towards the elucidation of this problem. We shall prove existence of two-soliton solutions for a class of relativistic field equations for a single, real, scalar, field in two dimensions (one space, one time).

Let $U(\rho), \rho \in \mathbf{R}$, be a real $C^{\infty}$ function with the following properties.

a) $U(\rho) \geqq 0$ everywhere.

b) There exists a (finite or infinite) set of points $P=\left\{\rho_{1}, \rho_{2}, \ldots\right\}$ such that

$$
\begin{aligned}
& U\left(\rho_{i}\right)=U^{\prime}\left(\rho_{i}\right)=0, U^{\prime \prime}\left(\rho_{i}\right)=\mu^{2}>0 \text { for } \rho_{i} \in P, \\
& U(\rho) \neq 0 \text { for } \rho \notin P .
\end{aligned}
$$

We call two elements $\rho_{i} \neq \rho_{j}$ of $P$ adjacent if no other element of $P$ lies between them.

Let

$H(x, t)=\frac{1}{2}\left[\partial_{t} \varphi(x, t)\right]^{2}+\frac{1}{2}\left[\partial_{x} \varphi(x, t)\right]^{2}+U(\varphi(x, t))$,

$\partial_{t}=\frac{\partial}{\partial t}, \partial_{x}=\frac{\partial}{\partial x}$, be the Hamiltonian density of a real, scalar, field in two-dimensional space-time. The corresponding field equation is

$\partial_{t}^{2} \varphi-\partial_{x}^{2} \varphi+U^{\prime}(\varphi)=0$.

Let $\rho_{1}, \rho_{2} \in P$ be two adjacent zeros of $U$. It is known (see Section II for a more detailed discussion) that there exists a static, real, solution $\varphi_{12}(x)$ of $(1.4)$ with

$$
\lim _{x \rightarrow-\infty} \varphi_{12}(x)=\rho_{1}, \lim _{x \rightarrow \infty} \varphi_{12}(x)=\rho_{2},
$$

and whose energy $H=\int d x H_{12}(x)$ is finite, where $H_{12}$ is the density (1.3) for $\varphi=\varphi_{12}$. The function $\varphi_{12}$ is $C^{\infty}$ and monotonic. It is uniquely determined up to translations of the argument, i.e. with $\varphi_{12}(x)$ also $\varphi_{12}(x-a), a \in \mathbf{R}$, is a solution of the desired type. In what follows we shall denote with $\varphi_{12}$ an arbitrary element, chosen once and for all, of this infinite family of solutions.

A soliton moving with velocity $v,|v|<c=1$, is obtained from $\varphi_{12}$ by Lorentz transformation:

$$
\varphi_{12}^{v}(x, t)=\varphi_{12}(\beta(x-v t)), \beta=\left(1-v^{2}\right)^{-1 / 2} .
$$

Clearly, this is again a solution of (1.4).

Let $\rho_{3} \in P$ be adjacent to $\rho_{2}$, so that either $\rho_{3}=\rho_{1}$, or $\rho_{2}$ lies between $\rho_{1}$ and 
$\rho_{3}$. We denote as $R$ the smallest closed interval of the $\rho$-axis containing $\rho_{1}, \rho_{2}, \rho_{3}$. In analogy to $\varphi_{12}^{v}$ we define $\varphi_{23}^{v}$. Let the velocities $v_{1}, v_{2}$, satisfy $-1<v_{2}<v_{1}<1$. We introduce the asymptotic two-soliton function with velocities $v_{i}$ :

$$
\begin{aligned}
& \varphi^{\text {in }}(x, t)=\varphi_{12}^{v_{1}}(x, t)+\varphi_{23}^{v_{2}}(x, t)-\rho_{2} . \\
& \varphi^{\text {in }} \text { satisfies } \\
& \lim _{x \rightarrow-\infty} \varphi^{\text {in }}(x, t)=\rho_{1}, \quad \lim _{x \rightarrow \infty} \varphi^{\text {in }}(x, t)=\rho_{3}, \\
& \lim _{t \rightarrow-\infty} \varphi^{\text {in }}(v t, t)=\rho_{2} \quad \text { if } v_{2}<v<v_{1} .
\end{aligned}
$$

Due to the monotonicity of $\varphi_{i j}$ we have $\varphi^{\text {in }} \in R$ for all $x$ and sufficiently small $t$. No restriction on $t$ is necessary in the case $\rho_{1} \neq \rho_{3}$.

The inequality $v_{1} \neq v_{2}$ is essential, since for $v_{1}=v_{2}$ the two solitons would always remain at a finite distance and never become independent. Actually we shall have to introduce the condition that $v_{1}$ and $v_{2}$ be "sufficiently different" from each other.

We can now state our main result:

Theorem. Let $U(\rho)$ and $\varphi^{\text {in }}(x, t)$ be the functions introduced above.

Then, for any non-negative integer $L$ and any two velocities $v_{1}, v_{2}$, sufficiently different from each other, there exist a positive number $\alpha$, a real number $T$, and a $C^{\infty}$ solution $\varphi(x, t)$ of $(1.4)$ defined for $t \leqq T$, such that

$$
\lim _{t \rightarrow-\infty} \sup _{x}\left|x^{l} e^{-\alpha t} D\left[\varphi(x, t)-\varphi^{\mathrm{in}}(x, t)\right]\right|=0
$$

for all derivatives $D=\partial_{x}^{r} \partial_{t}^{s}, r, s \geqq 0$, and all integers $l$ with $0 \leqq l \leqq L$.

Remarks. i) We only prove existence of the solution $\varphi$ in the half plane $t \leqq T$. However, according to results of Parenti, Strocchi, and Velo [8] this solution can be continued to a global solution (i.e. a solution defined everywhere), if $U$ is an entire function. Nothing is known about the behaviour of this solution for $t \rightarrow+\infty$.

ii) The choice $L \geqq 1$ ensures that the energy $\int d x H(x, t)$ of $\varphi$ is finite.

iii) Our proof of the Theorem uses quite crude methods. Hence our result is certainly not optimal. In particular, one would expect the Theorem to hold, possibly with a different form of the approach condition (1.9), for any $v_{1} \neq v_{2}$ irrespective of their closeness. This expectation is borne out by the known twosoliton solutions of the sine-Gordon equation.

iv) Our methods are in principle generalizable to more than two solitons, but may then lead to conditions on the velocities which cannot be satisfied.

The remainder of the paper is devoted to the proof of the Theorem. In Section II we discuss some relevant properties of one-soliton solutions. The proof proper will be given in Section III.

\section{One-Soliton Solutions}

Let us first recall some elementary facts about static soliton solutions (see e.g. 
Ref. [1]). A static solution $\varphi(x)$ of (1.4) satisfies the ordinary differential equation

$$
\varphi^{\prime \prime}(x)=U^{\prime}(\varphi(x)) \text {. }
$$

This is simply Newton's equation of motion for a one-dimensional particle moving in the potential $-U, x$ playing the role of time, $\varphi$ of position. Under the conditions of Section I we are interested in a particle moving in the potential trough limited by the two maxima of $-U(\varphi)$ at $\rho_{1}$ and $\rho_{2}$. A soliton corresponds to a solution with total energy zero:

$$
E=\frac{1}{2} \varphi^{\prime 2}-U(\varphi)=0 .
$$

Such solutions obviously exist. They are obtained by solving the simple differential equation

$$
\frac{d x}{d \varphi}=[2 U(\varphi)]^{-1 / 2}
$$

in the interval $I=] \rho_{1}, \rho_{2}$ [ and inverting the resulting function $x(\varphi)$ to get $\varphi(x)$. (This $\varphi$ was called $\varphi_{12}$ in Section I.) Note that $U(\varphi)>0$ in $I$, hence the right-hand side of (2.3) is regular and non-vanishing in $I$, so that $x(\varphi)$ is invertible. The monotonicity of the resulting $\varphi(x)$ is obvious. If $\rho_{1}<\rho_{2}$ we must take in (2.3) the positive square root, and $x(\varphi)$ and $\varphi(x)$ are increasing functions. If $\rho_{1}>\rho_{2}$ the negative root applies and $\varphi(x)$ is decreasing. Infinite differentiability of $x(\varphi)$, and therefore of $\varphi(x)$, follows easily from (2.3) and the properties of $U$. For $\varphi \rightarrow \rho_{1,2}$ we find $x \rightarrow \pm \infty$ due to the non-integrability of $U^{-1 / 2}$ at the boundaries of $I$. The solution $\varphi(x)$ is unique up to translations in $x$, the translation parameter being the one integration constant present in the general solution of the first order differential Equation (2.3). As mentioned in the introduction, we select an arbitrary solution for further consideration.

We need to know how fast $\varphi(x)$ approaches its limits $\rho_{1,2}$ if $x \rightarrow \pm \infty$ :

Lemma 1. Let $U(\rho)$ satisfy the conditions stated in Section $I$, and let $\varphi(x)$ be the soliton solution discussed above.

Then, for $|x| \rightarrow \infty$ the following functions: $\varphi(x)-\rho_{1}($ for $x \rightarrow-\infty), \varphi(x)-\rho_{2}$ $($ for $x \rightarrow \infty), U(\varphi(x)), U^{\prime}(\varphi(x)), U^{\prime \prime}(\varphi(x))-\mu^{2}$, as well as all their derivatives with respect to $x$, decrease of order $O\left(e^{-v|x|}\right)$ for every positive number $v<\mu$.

Proof of Lemma 1. In order to fix the ideas we consider the case $x \rightarrow+\infty$, $\rho_{1}<\rho_{2}$. Define $\psi(x)=\rho_{2}-\varphi(x)$, so that $\psi(x)>0$ is monotonically decreasing, and $\psi(x) \rightarrow 0$ for $x \rightarrow \infty$. Equation (2.2) becomes

$$
\psi^{\prime}(x)=-\left[2 U\left(\rho_{2}-\psi(x)\right)\right]^{1 / 2} .
$$

Let $v$ be a positive number smaller than $\mu$. Then we have by (1.2)

$$
U\left(\rho_{2}-\psi(x)\right) \geqq \frac{1}{2} v^{2}[\psi(x)]^{2}
$$

for $x$ sufficiently large, the minimal $x$ above which this inequality hold depending on $v$. Introducing this estimate into $(2.4)$ we find $\psi^{\prime}(x) \leqq-v \psi(x)$, hence $(\log \psi)^{\prime} \leqq$ $-v$, from which we obtain $\psi(x) \leqq$ const $\cdot e^{-v x}$ for $x$ sufficiently large. This proves the lemma for $\varphi(x)-\rho_{2}$. Writing $U(\varphi(x))=U\left(\rho_{2}-\psi(x)\right)$ and using the mean 
value theorem we find $U(\varphi(x))=O\left(e^{-v x}\right)$. The same argument applies to $U^{\prime}(\varphi)$ and $U^{\prime \prime}(\varphi)-\mu^{2}$. Equation (2.1) yields then the desired estimate also for $\psi^{\prime \prime}$, and by integration for $\psi^{\prime}$ (note that $\psi^{\prime} \rightarrow 0$ for $x \rightarrow \infty$ by (2.2)). Differentiation of (2.1) gives $\varphi^{\prime \prime \prime}=U^{\prime \prime}(\varphi) \cdot \varphi^{\prime}$, which again has the claimed behaviour. Continued differentiation gives the desired result for arbitrarily high derivatives of all the functions mentioned in the lemma.

It is clear how the statements of Lemma 1 transcribe to the functions $\varphi_{i j}^{v}$ and $\varphi^{\text {in }}$ of Section I. In Section III we shall need information on the function

$$
\begin{aligned}
u_{0}(x, t) & =-U^{\prime}\left(\varphi^{\mathrm{in}}\right)-\partial_{t}^{2} \varphi^{\mathrm{in}}+\partial_{x}^{2} \varphi^{\mathrm{in}} \\
& =U^{\prime}\left(\varphi_{12}\right)+U^{\prime}\left(\varphi_{23}\right)-U^{\prime}\left(\varphi_{12}+\varphi_{23}-\rho_{2}\right) .
\end{aligned}
$$

(For typographical convenience we omit the upper indices $v_{1,2}$.) Define $v=\frac{1}{2}\left(v_{1}+\right.$ $\left.v_{2}\right)$. Consider the half space $L=\{(x, t): x \leqq v t\}$. According to Lemma 1 the estimate

$$
\left|\varphi_{23}(x, t)-\rho_{2}\right| \leqq c \exp \left(-v \beta_{2}\left|x-v_{2} t\right|\right) \leqq c \exp \left(v \beta_{2}\left(v-v_{2}\right) t\right)
$$

holds in $L$ for sufficiently small $t$, with $\beta_{i}=\left(1-v_{i}^{2}\right)^{-1 / 2}$ and $c$ a positive constant. Introducing this estimate into (2.6) we find

$$
\begin{aligned}
u_{0}(x, t) & =U^{\prime}\left(\varphi_{12}\right)+U^{\prime}\left(\rho_{2}+O\left(e^{\alpha_{L} t}\right)\right)-U^{\prime}\left(\varphi_{12}+O\left(e^{\alpha_{L} t}\right)\right) \\
& =U^{\prime}\left(\varphi_{12}\right)+O\left(e^{\alpha_{L} t}\right)-U^{\prime}\left(\varphi_{12}\right)-O\left(e^{\alpha_{L} t}\right) \\
& =O\left(e^{\alpha_{L} t}\right)
\end{aligned}
$$

for $t \rightarrow-\infty$, uniformly in $x$, with $\alpha_{L}=\frac{1}{2} v \beta_{2}\left(v_{1}-v_{2}\right)$. In the same way we obtain in $R=\{(x, t): x \geqq v t\}$ the estimate $u_{0}(x, t)=O\left(\exp \left(\alpha_{R} t\right)\right)$, uniformly in $x$, with $\alpha_{R}=\frac{1}{2} v \beta_{1}\left(v_{1}-v_{2}\right)$. One convinces himself easily that the same sort of argument also applies to all derivatives of $u_{0}$ and their products with any positive power of $x$. In the latter case we must remember that Lemma 1 also holds with $v$ replaced by $v^{\prime}, v<v^{\prime}<\mu$.

Thus we have proved

\section{Lemma 2.}

$$
\left|x^{N} \partial_{t}^{r} \partial_{x}^{s} u_{0}(x, t)\right|=O\left(e^{\alpha t}\right)
$$

for $t \rightarrow-\infty$, uniformly in $x$, with

$$
\alpha=\frac{1}{2} v\left(v_{1}-v_{2}\right) \min \left(\beta_{1}, \beta_{2}\right)
$$

and $N, r, s$, any non-negative integers.

Note that $\alpha$ can be made arbitrarily large by choosing $v_{1}, v_{2}$, of different sign and letting both $\left|v_{i}\right|$ approach the limiting value 1 .

\section{Proof of the Theorem}

In order to solve Equation (1.4) with the initial conditions at $t=-\infty$ stated in the Theorem, we write $\varphi$ as

$$
\varphi(x, t)=\varphi^{\mathrm{in}}(x, t)+\delta(x, t) .
$$


Insertion of this ansatz into (1.4) yields

$$
\partial_{t}^{2} \delta-\partial_{x}^{2} \delta=-U^{\prime}\left(\varphi^{\mathrm{in}}+\delta\right)-\partial_{t}^{2} \varphi^{\mathrm{in}}+\partial_{x}^{2} \varphi^{\mathrm{in}},
$$

We wish to prove existence of a solution $\delta$ vanishing for $t \rightarrow-\infty$ in the sense indicated in the Theorem. Solving (3.2) for such a $\delta$ is equivalent to solving the integral equation

$$
\delta(x, t)=-\int d \xi d \eta D_{\mathrm{ret}}(x-\xi, t-\eta)\left[U^{\prime}\left(\varphi^{\mathrm{in}}(\xi, \eta)+\delta(\xi, \eta)\right)+\left(\partial_{\eta}^{2}-\partial_{\xi}^{2}\right) \varphi^{\mathrm{in}}(\xi, \eta)\right],
$$

where

$$
D_{\text {ret }}(x, t)=\frac{1}{4} \theta(t-x) \theta(t+x)
$$

is the retarded Green's function of the operator $\partial_{t}^{2}-\partial_{x}^{2}$. We shall use the notation

$$
D_{\text {ret }} * F=\int d \xi d \eta D_{\text {ret }}(x-\xi, t-\eta) F(\xi, \eta)
$$

for convolution integrals of the form occuring in (3.4).

Equation (3.3) can be written

$$
\delta=D_{\text {ret }} * u_{0}-D_{\text {ret }} *\left[U^{\prime}\left(\varphi^{\text {in }}+\delta\right)-U^{\prime}\left(\varphi^{\mathrm{in}}\right)\right],
$$

with $u_{0}$ as defined in (2.6). We solve this equation by iteration. Define

$$
\left.\begin{array}{rl}
\delta_{0}(x, t) & =0, \\
\delta_{1}(x, t) & =D_{\text {ret }} * u_{0}, \\
\delta_{n+1}(x, t) & =\delta_{1}-D_{\text {ret }} *\left[U^{\prime}\left(\varphi^{\text {in }}+\delta_{n}\right)-U^{\prime}\left(\varphi^{\text {in }}\right)\right], n>1 .
\end{array}\right\}
$$

We have then

$$
\delta_{n+1}-\delta_{n}=-D_{\text {ret }} *\left[U^{\prime}\left(\varphi^{\mathrm{in}}+\delta_{n}\right)-U^{\prime}\left(\varphi^{\mathrm{in}}+\delta_{n-1}\right)\right] .
$$

For any fixed, real, $T$ there exists, by Lemma 2 , a positive constant $M_{0}$ such that

$$
\left|u_{0}(x, t)\right| \leqq M_{0} e^{\alpha t}
$$

for $t<T . M_{0}$ remains bounded for $T \rightarrow-\infty$. Starting from this estimate we find by induction with respect to $n$ that the integrals in (3.7) exist and that there are positive constants $M_{n}, N_{n}$, depending on $T$ but remaining bounded for $T \rightarrow-\infty$, such that

$$
\left|\delta_{n}(x, t)\right| \leqq M_{n} e^{\alpha t},\left|\delta_{n+1}(x, t)-\delta_{n}(x, t)\right| \leqq N_{n} e^{\alpha t}
$$

for $t<T$.

The sequence $\delta_{n}$ converges for $n \rightarrow \infty$ towards a solution $\delta(x, t)$ of (3.6) if $\sum N_{n}<\infty$. We shall now prove that this is the case if $\alpha$ is sufficiently large and $T$ is sufficiently small. Let $a$ be an arbitrarily chosen positive number. Define

$$
R=\sup \left|\varphi^{\text {in }}(x, t)\right|<\infty, r=\sup _{|\rho| \leqq R+a}\left|U^{\prime \prime}(\rho)\right| .
$$

Assume $2 \alpha^{2}>r$. Since $\alpha$ becomes arbitrarily large for sufficiently different $v_{i}$ (see 
the remark after Lemma 2), there exist values of $v_{i}$ for which this assumption is satisfied. Finally, choose $T$ such that $M_{0}\left(2 \alpha^{2}-r\right)^{-1} e^{\alpha T}<a$. Under these assumptions we can prove the following results by induction with respect to $n$ :
i) $\left|\delta_{n}(x, t)\right|<a \quad$ in $t<T$,
ii) $N_{n}=\frac{r}{2 \alpha^{2}} N_{n-1}$,

hence

$$
N=\sum_{n=0}^{\infty} N_{n}=\frac{M_{0}}{2 \alpha^{2}-r}<\infty .
$$

From(3.7) and (3.9) we find easily that $N_{0}=M_{1}=M_{0}\left(2 \alpha^{2}\right)^{-1}<M_{0}\left(2 \alpha^{2}-r\right)^{-1}$. Hence (3.12) holds for $n=1$. Assume (3.12) to hold in all orders up to $n$. We have then

$$
\left|U^{\prime}\left(\varphi^{\text {in }}+\delta_{n}\right)-U^{\prime}\left(\varphi^{\text {in }}+\delta_{n-1}\right)\right| \leqq r\left|\delta_{n}-\delta_{n-1}\right| \leqq r N_{n-1} e^{\alpha t} .
$$

Inserting this in Equation (3.8) we find after an easy calculation the relation (3.13). Using this result we find, for $t<T$, that

$$
\left|\delta_{n+1}\right| \leqq \sum_{i=0}^{n}\left|\delta_{i+1}-\delta_{i}\right| \leqq \sum_{0}^{\infty} N_{i} e^{\alpha t} \leqq \frac{M_{0}}{2 \alpha^{2}-r} e^{\alpha t}<a:
$$

the estimate (3.12) holds also in order $n+1$.

From (3.14) we conclude uniform convergence of $\delta_{n}$ in $t<T$ towards a function $\delta(x, t)$ with $|\delta(x, t)| \leqq N e^{\alpha t}$. It is easy to see that $\delta$ solves the integral equation (3.3) and that $\varphi=\varphi^{\text {in }}+\delta$ satisfies (1.9) for $r=s=l=0$.

The case $l>0$ is treated by multiplying (3.7), (3.8), with $|x|^{l}$ and using on the right-hand side the identity $x^{l}=\sum_{k}\left(\begin{array}{l}l \\ k\end{array}\right) \xi^{k}(x-\xi)^{l-k}$. With the help of (2.8), written for $N=l, r=s=0$, we can then find estimates for $\left|x^{l} \delta_{n}\right|$ by a similar iterative method as for $\left|\delta_{n}\right|$ itself. For increasing $l$ the iteration possibly converges only under increasingly stringent conditions on $\alpha$ and $T$. This is the reason for introducing a finite upper limit $L$ for the allowed values of $l$. Validity of (1.9) for general $D$ follows by successive differentiation of (3.3), using on the right-hand side the information already obtained for $\delta$ itself and its lower derivatives. For instance, for the first $t$-derivative we find

$$
\begin{aligned}
\partial_{t} \delta(x, t)= & \frac{1}{4} \int d \xi d \eta[\delta(t-\eta-x+\xi) \theta(t-\eta+x-\xi)+\theta(t-\eta-x+\xi) \\
& \cdot \delta(t-\eta+x-\xi)]\left\{-U^{\prime}\left(\varphi^{\mathrm{in}}(\xi, \eta)+\delta(\xi, \eta)\right)-\left(\partial_{\eta}^{2}-\partial_{\xi}^{2}\right) \varphi^{\mathrm{in}}(\xi, \eta)\right\} .
\end{aligned}
$$

But the expression in the curly bracket is absolutely bounded by $\left|u_{0}\right|+r|\delta|$. From the known estimates for $u_{0}$ and $\delta$ we obtain exponential decrease of $\{\ldots\}$ for $t \rightarrow-\infty$, uniformly in $x$, and inserting this result in (3.15) we find existence and exponential decrease of $\partial_{t} \delta$. Repeated application of this method leads to $D$ 's of arbitrarily high order, and the same method applies if $l>0$. 
Since the second derivatives of $\delta$ exist and are well behaved, our solution $\delta$ of (3.3) solves also the original differential equation (3.2), hence $\varphi=\varphi^{\text {in }}+\delta$ solves (1.4).

Acknowledgment. I am greatly indebted to the referee for a suggestion leading to a considerable simplification in the proof of the main theorem, as well as to an extension of its range of validity.

\section{References}

1. Coleman, S. : Classical lumps and their quantum descendents. In: New phenomena in subnuclear physics, part A, (ed. A. Zichichi). New York and London: Plenum Press 1977

2. Rajaraman, R. : Phys. Rept. 21, 227 (1975)

3. Jackiw, R. : Rev. Mod. Phys. 49, 681 (1977)

4. Scott, A., Chu, F., McLaughlin, D.: Proc. IEEE 61, 1443 (1973)

5. Seeger, A., Donth, H., Kochendörfer, A. : Z. Physik 134, 173 (1953)

6. Perring, J. K., Skyrme, T. H. R. : Nucl. Phys. 31, 550 (1962)

7. Dashen, R., Hasslacher, B., Neveu, A. : Phys. Rev. D11, 3424 (1975)

8. Parenti, C., Strocchi, F., Velo, G. : Phys. Lett. 59B, 157 (1975); Ann. Scuola Norm. Sup. (Pisa), Ser. 4, 3, 443 (1976)

Communicated by J. Glimm

Received December 6, 1977 